\title{
Health literacy and knowledge related to tuberculosis among outpatients at a referral hospital in Lima, Peru
}

This article was published in the following Dove Medical Press journal: Research and Reports in Tropical Medicine

\author{
Rosalina Penaloza ${ }^{1-3}$ \\ Joanna Itzel Navarro $1,4,5$ \\ Pauline E Jolly' \\ Anna Junkins' \\ Carlos Seas ${ }^{6,7}$ \\ Larissa Otero ${ }^{6,7}$ \\ 'University of Alabama at Birmingham, \\ School of Public Health, Department \\ of Epidemiology, Birmingham, AL, USA; \\ ${ }^{2}$ David Geffen School of Medicine, \\ University of California, Los Angeles, \\ CA, USA; ${ }^{3}$ Charles R. Drew University, \\ College of Medicine and Science, Los \\ Angeles, CA, USA; ${ }^{4}$ Graduate School \\ of Education and Information Systems, \\ University of California, Los Angeles, \\ CA, USA; ${ }^{5}$ Henry Samueli School \\ of Engineering and Applied Science, \\ University of California, Los Angeles, \\ CA, USA; ${ }^{\prime}$ Instituto de Medicina \\ Tropical Alexander von Humboldt, \\ Universidad Peruana Cayetano \\ Heredia, Lima, Peru; ${ }^{7}$ Facultad \\ de Medicina Alberto Hurtado, \\ Universidad Peruana Cayetano \\ Heredia, Lima, Peru
}

Background: Tuberculosis (TB) case detection in Peru relies on passive case finding. This strategy relies on the assumption that the community is aware that a persistent cough or contact with a TB patient is an indication to seek formal health care. This study evaluated health literacy and TB knowledge among outpatients at Hospital Cayetano Heredia in Lima, Peru.

Methods: A cross-sectional survey was performed between June and August 2017. Data on sociodemographic factors, TB knowledge, and health literacy were collected, and bivariate and multivariate logistic regressions were performed to study the associations between variables. Results: The analysis included 272 participants; $57.7 \%$ knew someone who had TB and 9\% had TB in the past. A 2-week cough was reported as a TB symptom by 66 (24\%) participants. High TB knowledge was found among 149 (54.8\%) participants and high health literacy was found among $193(71.0 \%)$ participants. Health literacy and TB knowledge were not significantly associated ( $\mathrm{OR}=0.9 ; 95 \% \mathrm{CI} 0.5-1.5)$. After controlling for sex, age, district, education, health insurance, frequency of hospital visits, and previous TB diagnosis, high TB knowledge was associated with knowing someone with $\mathrm{TB}(\mathrm{aOR}=2.7$; 95\% CI 1.6-4.7) and inversely associated with being a public transport driver $(\mathrm{aOR}=0.2 ; 95 \% \mathrm{CI} 0.05-0.9)$. Not living in poverty was the single factor associated with high health literacy (aOR=3.8; 95\% CI 1.6-8.9).

Conclusion: Although TB knowledge was fair, $30 \%$ did not know that cough is a symptom of $\mathrm{TB}$ and $>70 \%$ did not know being in contact with a TB patient is a risk factor for TB. Tailoring educational strategies to at-risk groups may enhance passive case detection especially among transport workers and TB contacts in Lima, Peru.

Keywords: health literacy, tuberculosis, tuberculosis knowledge, Peru

\section{Description of significance}

Peru has one of the highest burdens of tuberculosis (TB) in the Americas. The annual incidence is 117 cases per 100,000 population (2016). Lima, the capital of Peru, accounts for approximately $58 \%$ of the country's TB cases. As in most countries with a high TB burden, TB case detection in Peru relies on passive case finding. This strategy relies on the assumption that the community is aware that a persistent cough or contact with a TB patient is an indication to seek formal health care. There are occasional educational campaigns, but the level of knowledge and awareness is not clear. Furthermore, in Latin America, very few studies have evaluated level of health literacy and its impacts. In Peru, only one study of gynecological patients evaluated health literacy and no study to date has examined the relationship between health literacy and TB knowledge. We believe that health literacy plays an important role in the acquisition of skills to navigate
Correspondence: Larissa Otero Instituto de Medicina Tropical Alexander von Humboldt, Universidad Peruana Cayetano Heredia, Av. Honorio Delgado 430, San Martín de Porres, Lima, Peru Email larissaotero@gmail.com
Research and Reports in Tropical Medicine 2019:10 I-10

(c) \& 2019 Penaloza et al. This work is published and licensed by Dove Medical Press Limited. The full terms of this license are available at https://www.dovepress.com/term

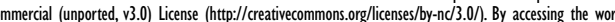
you herby accept the Terms. Non-commercial uses of the work are permitted without any further permission from Dove Medical Press Limited, provided the work is properly attributed. For permission for commercial use of this work, please see paragraphs 4.2 and 5 of our Tems (https://www.doveperess.com/terms.php. 
the health care systems and understand and implement health information to prevent the spread of diseases like TB. Our goal was to ascertain knowledge gaps in key areas relating to TB, to identify and prioritize subgroups for intervention, and to provide insight for policy and community interventions to increase health literacy.

\section{Introduction}

Health literacy, as defined by the US National Library of Medicine, is a set of knowledge and skills that determines one's ability to obtain, understand, and apply health information in ways that promote good health. ${ }^{1,2}$ Consistent findings suggest a causal relationship between limited health literacy and adverse health outcomes, including higher rates of emergency room visits, inefficient use of medical services, and poor management of chronic diseases. ${ }^{3-10}$ The National Assessment of Adult Literacy found that among 19,000 US adults, $88 \%$ did not have the health literacy skills needed to manage all the demands of the health care system. ${ }^{11}$ In Latin America, very few studies have evaluated health literacy and its impacts; studies of outpatients in South America found that around a third had limited health literacy. ${ }^{12,13}$ In Peru, only one study of gynecological patients evaluated health literacy and found that $43 \%$ of patients had low health literacy. ${ }^{14} \mathrm{We}$ believe that health literacy plays an important role in the acquisition of skills to navigate the health care system and understand and implement health information to prevent spread of diseases like tuberculosis (TB), which despite having successful diagnostics and treatment, in the 21 st century is the top infectious disease killer worldwide. ${ }^{15}$ In Peru, no study to date has examined the relationship between health literacy and TB knowledge.

Peru has one of the highest burdens of TB in the Americas. The annual incidence is 117 cases per 100,000 population (2016). ${ }^{15,16}$ In Peru, coinfection with HIV, undernutrition, crowded living conditions, and poor socioeconomic status are known risk factors for the spread of TB. As in most countries with high TB burden, TB case detection in Peru depends heavily on passive case finding. This strategy relies on the assumption that the community is aware of the risks and signs that merit an evaluation of TB including a persistent cough or contact with a TB patient. There are occasional educational campaigns, but level of knowledge and health literacy is not clear. ${ }^{17}$ This study aims to assess health literacy and TB knowledge among outpatients at a referral hospital in Lima, Peru. Our goal was to ascertain knowledge gaps in key areas relating to $\mathrm{TB}$, to identify and prioritize subgroups for intervention, and to provide insight for policy and community interventions to increase health literacy.

\section{Materials and methods Study design and setting}

We conducted an observational cross-sectional study using a survey to measure sociodemographic factors, TB knowledge, and health literacy. The study was conducted at Hospital Cayetano Heredia in Lima, Peru, from June to August 2017. This hospital is a level-three health care facility and serves 2,489,295 residents from nine districts in Lima. Lima, the capital of Peru, accounts for approximately 58\% of the country's TB cases. ${ }^{18,19}$

\section{Study procedures, questionnaire design, and data collection}

From Monday to Saturday, during clinic hours, two trained researchers (RP, JIN) approached adults ( $\geq 18$ years) sitting in the waiting areas of outpatient services including gynecology and obstetrics, family medicine, geriatrics, nephrology, psychology, hematology and oncology, infectious diseases, and pulmonology. Adults, chosen at random to avoid selection bias, were invited to participate, and those consenting were enrolled. Survey items were read out loud to the participant, and their responses were recorded in a database designed on Qualtrics (Qualtrics, Provo, UT, USA) using iPads (Apple Corp., Cupertino, CA, USA). The survey had three sections: TB knowledge, sociodemographic characteristics, and health literacy. We pilot tested the first two sections among 88 participants to address ambiguity and ensure their appropriateness.

\section{TB knowledge}

Participants were asked nine questions on signs and symptoms of TB, four on transmission and risk factors, two on diagnosis and treatment, and one on the perception of TB burden in Peru. For these items, spontaneous responses were first recorded, and then participants were prompted to respond to predetermined options. They were also asked about previous TB diagnosis and whether they knew someone who had had TB. A multiple-choice question elicited preferences on how they would like to receive information on TB.

\section{Sociodemographic data}

Sociodemographic data - age, sex, residence, occupation, education, type of health insurance, frequency of medical visits, and socioeconomic status - were collected. Socioeconomic status was measured using a 17-item tool, SISFOH, 
validated by the Ministry of Finance in $\operatorname{Peru}^{20}$ that includes type and material of the dwelling, access to basic services such as water and electricity, ownership of household items including a television, cell phone, radio, and level of education of the head of the household, among other items.

\section{Health literacy}

We used the 18-item Short Assessment of Health Literacy Spanish (SAHL-S) tool, based on its demonstrated validity and reliability in Spanish-speaking populations. SAHL-S and the longer 50-item SAHLSA-50 were developed by the same researchers using the same methods and both aim to test an individual's word recognition, reading comprehension, and ability to make word associations in a health context. ${ }^{21}$ Participants were shown an index card with three words: the main health term, one associated word, and one distracter, and were asked to read each of the 18 health terms aloud and then choose a term associated in meaning.

\section{Data management and analysis}

Data were exported from Qualtrics to R Project (R Core Team, Vienna, Austria) for data cleaning. Data coding and analysis were performed in STATA/IC 15.0 (StataCorp, College Station, TX, USA).

For the univariate description of TB knowledge and sociodemographic characteristics, we calculated frequencies and percentages for categorical variables. We analyzed age as a continuous variable, calculated its median and interquartile ranges (IQRs), and categorized it into four age groups based on the quartiles. We calculated a TB knowledge score by assigning two points for correct spontaneous responses and one for correct elicited responses on items in TB risk factors, signs, and symptoms. Incorrect responses, either spontaneous or elicited, received zero points. The rest of the items received one point for correct responses and zero for incorrect responses. The highest score possible was 30 . We categorized TB knowledge into low and high categories using the median as the cutoff; thus, participants scoring above the median were considered to have high TB knowledge.

To calculate socioeconomic status, each item response was matched to a numerical value assigned by the SISFOH scale and the total value was used to categorize individuals into living in extreme poverty, living in poverty, or not living in poverty.

The health literacy score was determined by assigning one point to each of the 18 health terms if the participant could correctly pronounce and associate the health term. The highest score possible as dictated by the SAHL-S was
18 points; participants were categorized as having low health literacy if they scored $\leq 14$ points and high health literacy if they scored $\geq 15$ points.

In the bivariate analysis, we tested the direction and strength of association between the following: 1) sociodemographic factors and health literacy, 2) sociodemographic factors and TB knowledge, and 3) health literacy and TB knowledge. We report absolute and relative frequencies as well as crude OR and 95\% CI calculated by logistic regression. We performed multivariable analysis using backward logistic regression to adjust the crude OR for potential confounders. The multivariable analysis included variables that had a significance value of $P<0.2$ in the bivariate analysis. Variables with the weakest association were taken out individually, and the models were compared until a model was found to be significantly different $(P<0.05)$ when a variable was taken out of the model.

\section{Ethical considerations}

This study was approved by the Institutional Review Boards of Hospital Cayetano Heredia, University of Alabama at Birmingham in the United States, and Universidad Peruana Cayetano Heredia. Written consent was obtained. An informative TB leaflet developed by the Peruvian Ministry of Health was given to each participant, as well as a 10 Peruvian Nuevos Soles (USD \$3.30) reimbursement as a token of appreciation.

\section{Results}

\section{Participant characteristics}

Two hundred seventy-two participants were included in the analysis (Figure 1), of which 221 (81.3\%) were patients; the remainder were family members or friends accompanying the patient. The median age was 39 years old (IQR=29-52). The majority were female $(66.2 \%) ; 17.7 \%$ attained education from a university, $14.7 \%$ from a technical institution, $46 \%$ received secondary education, and $21.7 \%$ received primary or no education. Most participants reported an occupation (93.4\%); the most common occupations were homemaking (33.5\%), others $(29.4 \%)$ including administration and seamstress, commerce (8.8\%), and student (8.8\%). About 10.3\% of participants experienced some poverty. Over $68 \%$ were recipients of the state health insurance (SIS) and $11 \%$ were uninsured. The remainder received social security (EsSalud; $16.9 \%$ ) or had a private insurance $(3.3 \%)$. About a quarter $(26.5 \%)$ of participants reported visiting the hospital at least once a month; $65.1 \%$ of participants perceived their health status as "good" or "excellent" at the time of the interview (Table 1). 


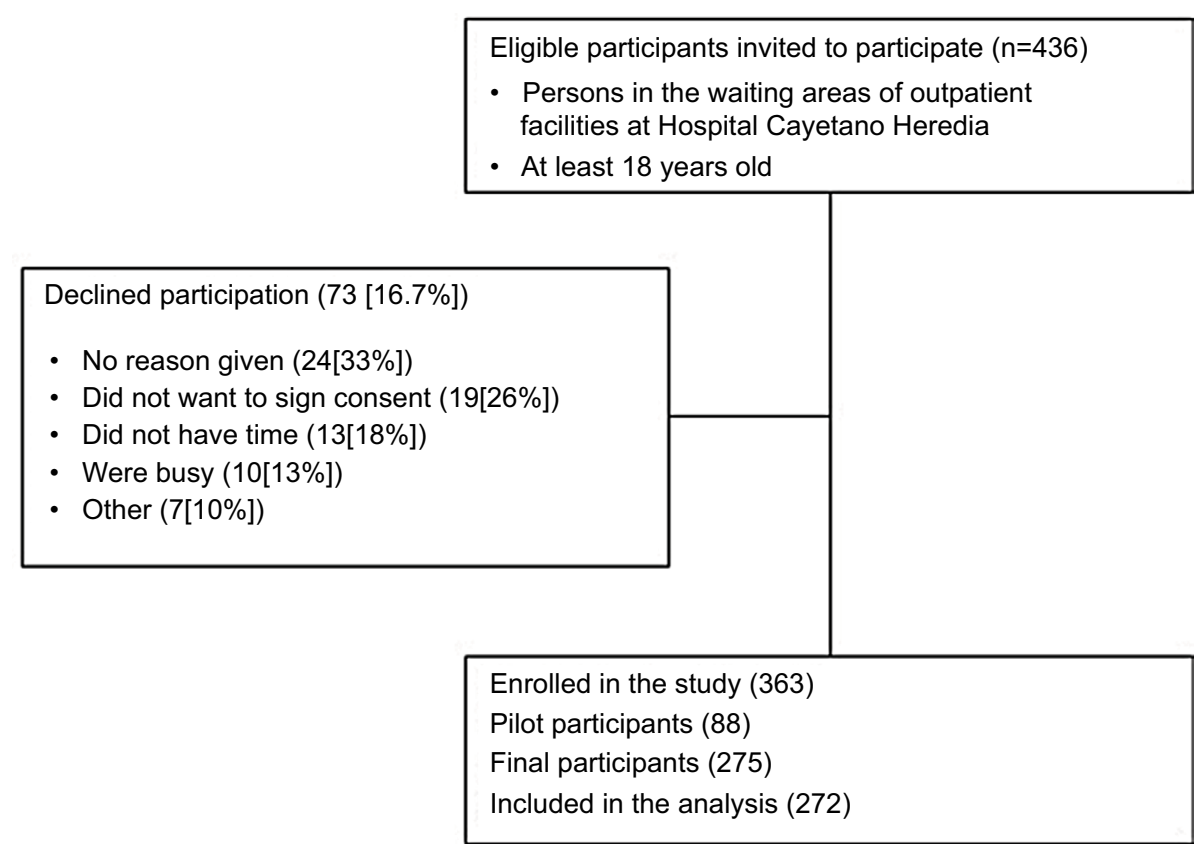

Figure I Study population, among outpatients attending Hospital Cayetano Heredia, June-August, 20 I7.

\section{TB knowledge}

Most participants, 269 (98.9\%), were aware of TB, and 193 (71.0\%) knew of TB's prevalence in Peru. Only 79 (29.0\%) thought there were no or "very few" TB cases or did not know if TB was prevalent in Peru. One hundred fifty-seven (57.7\%) participants knew someone who had TB and $23(9 \%)$ had a previous TB diagnosis. More than half, 155 (57.0\%), reported ever being tested for TB.

The median score in the overall TB knowledge assessment was 18 (IQR=16-20); 149 (54.8\%) of the participants had high TB knowledge while 123 (45.2\%) had low TB knowledge. Eleven (9.7\%) participants did not know how a person becomes infected or becomes at risk of acquiring TB. Participant responses to questions related to TB transmission and risk factors, signs, and symptoms are shown in Table 2 .

When asked if open or closed windows in public transportation was better for their health, 205 (75.4\%) of the participants correctly identified "open for ventilation", while 46 (16.9\%) indicated "closed to avoid catching a cold" or did not know. Most participants, 262 (96.3\%), indicated they would go to a formal health facility (doctor, hospital, health center) if they had any symptoms of TB. Two hundred seven participants (76.1\%) identified medication prescribed by a doctor as the cure for TB; 66 (24.3\%) said better nutrition, and $55(20.2 \%)$ said traditional medicine, no cure, or they did not know. Most participants, 178 (75.1\%), knew that TB treatment is free in Peru; 29 (12.2\%) said partially free or not free, and 30 (12.7\%) did not know.

When asked how they would like to receive information on TB (responses were not mutually exclusive), participants preferred health staff talks in the waiting areas (180 [66.2\%]), followed by television (173 [63.6\%]), medical staff during consultation (156 [57.4\%]), and social media (148 [54.4\%]).

\section{Health literacy}

The median score based on the SAHL-S instrument was 16 (IQR=14-17); 193 (71.0\%) of the participants had high health literacy and $79(29.0 \%)$ had low health literacy. Health literacy and TB knowledge were not significantly associated $(\mathrm{OR}=0.9 ; 95 \% \mathrm{CI} 0.5-1.5)$ in the bivariate analysis (Table 3$)$.

\section{Determinants of TB knowledge and health literacy}

The only determinants of TB knowledge were occupation (homemakers were $80 \%$ more likely than drivers to have high TB knowledge) and knowing someone with TB (participants were 2.7 times [OR=2.7; 95\% CI 1.6-4.7] more likely to have high TB knowledge if they knew someone with TB).

In the multivariate analysis, socioeconomic status was the single factor that remained associated with health literacy 
Table I Characteristics of participants enrolled in the study, Hospital Cayetano Heredia, June-August, 2017

\begin{tabular}{|c|c|}
\hline Participant characteristics $(\mathrm{N}=\mathbf{2 7 2})$ & n (\%) \\
\hline \multicolumn{2}{|l|}{ Age } \\
\hline Median (IQR) & $39(29-52)$ \\
\hline $18-32$ & $98(36.0)$ \\
\hline $33-47$ & $85(31.3)$ \\
\hline $48-62$ & $67(24.6)$ \\
\hline$\geq 63$ & $22(8.1)$ \\
\hline \multicolumn{2}{|l|}{ Gender } \\
\hline Female & $180(66.2)$ \\
\hline Male & $92(33.8)$ \\
\hline \multicolumn{2}{|l|}{ District of residence } \\
\hline San Martín de Porres & $83(30.5)$ \\
\hline Los Olivos & $58(21.3)$ \\
\hline Independencia & $34(12.5)$ \\
\hline Comas & $19(7.0)$ \\
\hline Other $^{a}$ & $78(28.7)$ \\
\hline \multicolumn{2}{|l|}{ Highest education attained } \\
\hline None/primary & $59(21.7)$ \\
\hline Secondary & $125(46.0)$ \\
\hline Technical & $40(14.7)$ \\
\hline University & $48(17.6)$ \\
\hline \multicolumn{2}{|l|}{ Occupation } \\
\hline None & $18(6.6)$ \\
\hline Homemaker & $91(33.5)$ \\
\hline Commerce & $24(8.8)$ \\
\hline Student & $24(8.8)$ \\
\hline Driver/collector & $14(5.2)$ \\
\hline Construction & II (4.0) \\
\hline Health care workers & $10(3.7)$ \\
\hline Other $^{\mathrm{b}}$ & $80(29.4)$ \\
\hline \multicolumn{2}{|l|}{ Socioeconomic status } \\
\hline No poverty & $244(89.7)$ \\
\hline Poverty & $28(10.3)$ \\
\hline \multicolumn{2}{|l|}{ Health insurance } \\
\hline None & $30(11.0)$ \\
\hline State health insurance & $187(68.8)$ \\
\hline Social security (EsSalud) & $46(16.9)$ \\
\hline Other/Private & $9(3.3)$ \\
\hline \multicolumn{2}{|c|}{ Frequency of medical visits to the hospital } \\
\hline At least once a month & $72(26.5)$ \\
\hline Every 3 months & $62(22.8)$ \\
\hline Twice a year & $55(20.2)$ \\
\hline Once a year or less & $83(30.5)$ \\
\hline \multicolumn{2}{|c|}{$\begin{array}{l}\text { Self-reported perception of health status } \\
\text { at the moment of the interview }\end{array}$} \\
\hline "Good" or "excellent" & $177(65.0)$ \\
\hline "Bad" or "really bad" & $23(8.5)$ \\
\hline "Regular" & $72(26.5)$ \\
\hline
\end{tabular}

Notes: "Other" includes other less reported districts including Ate, San Juan de

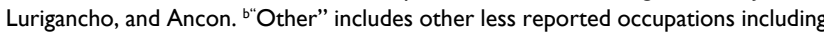
secretary, seamstress, and administrative work.

Abbreviation: IQR, interquartile range.

(Table 4); participants who were not living in poverty were almost four times (OR=3.8; 95\% CI 1.6-8.9) more likely to have high health literacy compared with those living in poverty.
Table 2 TB knowledge among outpatients attending Hospital Cayetano Heredia, June-August, 2017

\begin{tabular}{|c|c|c|}
\hline TB knowledge $(\mathrm{N}=272)$ & $\begin{array}{l}\text { Spontaneous } \\
\text { n (\%) }\end{array}$ & $\begin{array}{l}\text { Elicited } \\
\text { n (\%) }\end{array}$ \\
\hline \multicolumn{3}{|c|}{$\begin{array}{l}\text { How do you think one gets } \\
\text { tuberculosis? }\end{array}$} \\
\hline \multicolumn{3}{|c|}{$\begin{array}{l}\text { Through the air, when someone with TB coughs/ } \\
\text { sneezes? }\end{array}$} \\
\hline Yes & 89 (32.7) & $159(58.5)$ \\
\hline No & $\mathrm{NA}^{\mathrm{b}}$ & $24(8.8)$ \\
\hline \multicolumn{3}{|l|}{ From malnutrition? } \\
\hline Yes & $128(47.1)$ & $123(45.2)$ \\
\hline No & NA & $21(7.7)$ \\
\hline \multicolumn{3}{|l|}{$\begin{array}{l}\text { From being in contact with an } \\
\text { untreated person? }\end{array}$} \\
\hline Yes & $76(27.9)$ & $156(57.4)$ \\
\hline No & NA & $40(14.7)$ \\
\hline \multicolumn{3}{|c|}{ From being on public transport? } \\
\hline Yes & $13(4.8)$ & $229(84.2)$ \\
\hline No & NA & $30(11.0)$ \\
\hline \multicolumn{3}{|c|}{$\begin{array}{l}\text { What are the symptoms of } \\
\text { tuberculosis?c }\end{array}$} \\
\hline \multicolumn{3}{|l|}{ Cough? } \\
\hline Yes & $191(70.2)$ & $7 I(26.1)$ \\
\hline No & NA & $10(3.7)$ \\
\hline \multicolumn{3}{|l|}{ Fatigue and weakness? } \\
\hline Yes & 48 (I7.7) & $203(74.6)$ \\
\hline No & NA & \begin{tabular}{|l|}
$21(7.7)$ \\
\end{tabular} \\
\hline \multicolumn{3}{|l|}{ Weight loss? } \\
\hline Yes & $74(27.2)$ & $183(67.3)$ \\
\hline No & NA & $15(5.5)$ \\
\hline \multicolumn{3}{|l|}{ Fever? } \\
\hline Yes & $92(33.8)$ & $117(43.0)$ \\
\hline No & NA & $63(23.2)$ \\
\hline \multicolumn{3}{|c|}{ Cough for two or more weeks? } \\
\hline Yes & $66(24.3)$ & $188(69.1)$ \\
\hline No & NA & $18(6.6)$ \\
\hline \multicolumn{3}{|l|}{ Coughing sputum with blood? } \\
\hline Yes & $35(12.9)$ & $158(58.1)$ \\
\hline No & NA & $79(29.0)$ \\
\hline \multicolumn{3}{|l|}{ Shortness of breath? } \\
\hline Yes & $7(2.6)$ & $185(68.0)$ \\
\hline No & $\mathrm{NA}$ & $80(29.4)$ \\
\hline \multicolumn{3}{|l|}{ Night sweating? } \\
\hline Yes & $36(13.2)$ & I54(56.6) \\
\hline No & $\mathrm{NA}$ & $82(30.2)$ \\
\hline \multicolumn{3}{|l|}{ Chest pain? } \\
\hline Yes & II (4.0) & $161(59.2)$ \\
\hline No & NA & $100(36.8)$ \\
\hline
\end{tabular}

Notes: "Other" spontaneous responses not reported in the table were "sharing utensils" (40 [35.2\%]) and "low defenses" (14 [12.3\%]). b"No" is not a possible spontaneous response. "Other" symptoms reported spontaneously by the participants were "poor appetite" (3। [27.3\%]) and "backache" (18 [15.8\%]). Fifteen (13.3\%) did not know any symptoms of TB.

Abbreviation: TB, tuberculosis.

\section{Discussion}

We found that most outpatients attending a referral hospital in northeastern Lima had high health literacy and only fair knowledge of TB. Health literacy and TB knowledge were 
Table 3 Bivariate and multivariate analyses of participant characteristics and tuberculosis knowledge among outpatients attending Hospital Cayetano Heredia, June-August, 2017

\begin{tabular}{|c|c|c|c|c|c|c|}
\hline $\begin{array}{l}\text { Participant characteristics } \\
(\mathrm{N}=272)\end{array}$ & $\begin{array}{l}\text { High TB } \\
\text { knowledge n (\%) }\end{array}$ & $\begin{array}{l}\text { Low TB } \\
\text { knowledge } n(\%)\end{array}$ & $\begin{array}{l}\text { Crude OR } \\
(95 \% \mathrm{Cl})\end{array}$ & $P$-value & $\begin{array}{l}\text { Adjusted OR } \\
(95 \% \mathrm{Cl})\end{array}$ & $\begin{array}{l}P \text {-value } \\
(<0.05)\end{array}$ \\
\hline \multicolumn{7}{|l|}{ Type of participant } \\
\hline Patient & $124(56.1)$ & $97(43.9)$ & $1.3(0.7-2.4)$ & 0.36 & & \\
\hline Family/friend & $25(49.0)$ & $26(51.0)$ & $\mathrm{I}$ & & & \\
\hline \multicolumn{7}{|l|}{ Age group } \\
\hline $18-32$ & $42(42.9)$ & $56(57.1)$ & I & - & I & - \\
\hline $33-47$ & $53(62.4)$ & $32(37.7)$ & $2.2(1.2-4.0)^{\mathrm{a}}$ & $0.0 \mathrm{I}^{\mathrm{a}}$ & $1.7(0.8-3.5)$ & 0.16 \\
\hline $48-62$ & $42(62.7)$ & $25(37.3)$ & $2.2(1.2-4.2)^{\mathrm{a}}$ & $0.0 \mathrm{I}^{\mathrm{a}}$ & $1.7(0.8-3.8)$ & 0.18 \\
\hline$\geq 63$ & $12(54.6)$ & $10(45.5)$ & $1.6(0.6-4.1)$ & 0.32 & $1.4(0.5-4.1)$ & 0.54 \\
\hline \multicolumn{7}{|l|}{ Gender } \\
\hline Female & $104(57.8)$ & $76(42.2)$ & $1.4(0.9-2.4)^{\mathrm{a}}$ & $0.17^{\mathrm{a}}$ & - & \\
\hline Male & $45(48.9)$ & $47(51.1)$ & 1 & - & & \\
\hline \multicolumn{7}{|c|}{ District where the participant lives } \\
\hline San Martin de Porres & $41(49.4)$ & $42(50.6)$ & $0.5(0.2-1.3)^{\mathrm{a}}$ & $0.14^{\mathrm{a}}$ & - & \\
\hline Los Olivos & $35(60.3)$ & $23(39.7)$ & $0.7(0.2-2.1)$ & 0.53 & & \\
\hline Independencia & $18(52.9)$ & $16(47.1)$ & $0.5(0.2-1.7)$ & 0.28 & & \\
\hline Comas & $13(68.4)$ & $6(31.6)$ & $\mathrm{I}$ & - & & \\
\hline Other & $42(53.9)$ & $36(46.2)$ & $\mathrm{n} / \mathrm{a}$ & & & \\
\hline \multicolumn{2}{|l|}{ Highest education attained } & & & & - & \\
\hline None/primary & $33(55.9)$ & $26(44.1)$ & $1.2(0.7-2.3)$ & 0.48 & & \\
\hline Secondary & $63(50.4)$ & $62(49.6)$ & $\mathrm{I}$ & - & & \\
\hline Technical & $24(60.0)$ & $16(40.0)$ & $1.5(0.7-3.0)$ & 0.29 & & \\
\hline University & $29(60.4)$ & $19(39.6)$ & $1.5(0.8-3.0)$ & 0.24 & & \\
\hline \multicolumn{7}{|l|}{ Occupation } \\
\hline None & $10(55.6)$ & $8(44.4)$ & I.I (0.4-3.2) & 0.79 & $1.2(0.4-3.5)$ & 0.74 \\
\hline Homemaker & $49(52.1)$ & $45(47.9)$ & I & - & I & - \\
\hline Commerce & $15(62.5)$ & $9(37.5)$ & $1.5(0.6-3.8)$ & 0.37 & $1.2(0.4-3.2)$ & 0.73 \\
\hline Student & II (39.3) & $17(60.7)$ & $0.6(0.3-1.4)$ & 0.24 & $1.0(0.4-2.8)$ & 0.97 \\
\hline Driver/collector & $3(2 \mid .4)$ & II (78.6) & $0.3(0.1-1.0)^{\mathrm{a}}$ & $0.04^{a}$ & $0.2(0.05-0.9)$ & 0.03 \\
\hline Construction & $8(72.7)$ & $3(27.3)$ & $2.4(0.6-9.8)$ & 0.21 & $3.0(0.7-13.0)$ & 0.15 \\
\hline Hospital workers & $4(36.4)$ & $7(63.6)$ & $0.5(0.1-1.9)$ & 0.33 & $0.9(0.2-3.7)$ & 0.91 \\
\hline Other & $49(68.1)$ & $23(31.9)$ & $\mathrm{n} / \mathrm{a}$ & & & \\
\hline \multicolumn{7}{|l|}{ Socioeconomic status } \\
\hline Not living in poverty & $134(54.9)$ & $110(45.1)$ & $\mathrm{I} . \mathrm{I}(0.5-2.3)$ & 0.89 & & \\
\hline Living in poverty & $15(53.6)$ & $13(46.4)$ & 1 & - & & \\
\hline \multicolumn{7}{|l|}{ Health Insurance } \\
\hline None & $20(66.7)$ & $10(33.3)$ & I & - & I & - \\
\hline State health insurance & $95(50.8)$ & $92(49.2)$ & $0.5(0.2-1.2)^{\mathrm{a}}$ & $0.11^{\mathrm{a}}$ & $0.5(0.2-1.2)$ & 0.11 \\
\hline Social security insurance & $26(56.5)$ & $20(43.5)$ & $0.7(0.2-1.7)$ & 0.38 & $0.8(0.3-2.2)$ & 0.64 \\
\hline Other/Private & $8(88.9)$ & $\mathrm{I}(\mathrm{II} . \mathrm{I})$ & $4(0.4-36.6)$ & 0.22 & $4.6(0.5-47.3)$ & 0.20 \\
\hline \multicolumn{7}{|l|}{ Frequency of hospital visits } \\
\hline At least monthly & $40(55.6)$ & $32(44.4)$ & $1.1(0.6-2.1)$ & 0.75 & & \\
\hline Every 3 months & $33(53.2)$ & $29(46.8)$ & $1.0(0.5-2.0)$ & 0.98 & & \\
\hline Twice a year & $32(58.2)$ & $23(41.8)$ & $1.2(0.6-2.5)$ & 0.55 & & \\
\hline Once a year or less & $44(53.0)$ & $39(47.0)$ & 1 & - & & \\
\hline \multicolumn{7}{|c|}{ Self-reported perception of health status } \\
\hline Good/excellent & $98(55.4)$ & $79(44.6)$ & I & - & & \\
\hline Bad/really bad & $13(56.5)$ & $10(43.5)$ & $1.0(0.4-2.5)$ & 0.92 & & \\
\hline Regular & $38(52.8)$ & $34(47.2)$ & $0.9(0.5-1.6)$ & 0.71 & & \\
\hline \multicolumn{7}{|l|}{ Previous TB diagnosis } \\
\hline Yes & $16(69.6)$ & $7(30.4)$ & $2.0(0.8-5.0)^{\mathrm{a}}$ & $0.14^{a}$ & & \\
\hline No & $133(53.4)$ & $116(46.6)$ & I & - & & \\
\hline \multicolumn{7}{|c|}{ Knowing someone who had TB ever } \\
\hline Yes & $104(66.2)$ & $53(33.8)$ & $3.1(1.9-5.0)^{\mathrm{a}}$ & $0.00^{\mathrm{a}}$ & $2.7(1.6-4.7)$ & 0.00 \\
\hline No & $45(39.1)$ & $70(60.9)$ & I & - & I & - \\
\hline \multicolumn{7}{|l|}{ Health Literacy } \\
\hline High health literacy & $104(53.9)$ & $89(46.1)$ & $0.9(0.5-1.5)$ & 0.64 & & \\
\hline Low health literacy & $45(57.0)$ & $34(43.0)$ & 1 & - & & \\
\hline
\end{tabular}

Notes: ${ }^{2}$ Variables with a $P<0.2$ in the bivariate analysis were included in the multivariate analysis. Adjusted $\mathrm{OR}$ and $95 \% \mathrm{Cl}$ are reported only for variables that remained significant or acted as confounders in the final multivariate model. Bold $p$-values $<0.05$ are statistically significant.

Abbreviation: TB, tuberculosis. 
Table 4 Bivariate and multivariate analyses of participant characteristics and health literacy among outpatients attending Hospital Cayetano Heredia, June-August 2017

\begin{tabular}{|c|c|c|c|c|c|c|}
\hline $\begin{array}{l}\text { Participant } \\
\text { characteristics }(\mathrm{N}=272)\end{array}$ & $\begin{array}{l}\text { High health } \\
\text { literacy n (\%) }\end{array}$ & $\begin{array}{l}\text { Low health } \\
\text { literacy n (\%) }\end{array}$ & $\begin{array}{l}\text { Crude OR } \\
(95 \% \mathrm{Cl})\end{array}$ & $P$-value & $\begin{array}{l}\text { Adjusted OR } \\
(95 \% \mathrm{Cl})\end{array}$ & $P$-value \\
\hline \multicolumn{7}{|l|}{ Type of participant } \\
\hline Patient & $164(74.2)$ & $57(25.8)$ & $2.2(I .2-4.1)^{\mathrm{a}}$ & $0.02^{\mathrm{a}}$ & - & \\
\hline Family/friend & $29(56.9)$ & $22(43.1)$ & $\mathrm{I}$ & - & & \\
\hline Age group & & & & & - & \\
\hline $18-32$ & $70(71.4)$ & $28(28.6)$ & I & - & & \\
\hline $33-47$ & $62(72.9)$ & $23(27.1)$ & I.I $(0.6-2.1)$ & 0.82 & & \\
\hline $48-62$ & 45 (67.2) & $22(32.8)$ & $0.8(0.4-1.6)$ & 0.56 & & \\
\hline$\geq 63$ & $16(72.7)$ & $6(27.3)$ & I.I (0.4-3.0) & 0.90 & & \\
\hline \multicolumn{7}{|l|}{ Gender } \\
\hline Female & $129(71.7)$ & $51(28.3)$ & I.I $(0.6-1.9)$ & 0.72 & & \\
\hline Male & $64(69.6)$ & $28(30.4)$ & $\mathrm{I}$ & - & & \\
\hline \multicolumn{7}{|l|}{ District of residence } \\
\hline San Martín de Porres & $58(69.9)$ & $25(30.1)$ & I.I (0.4-3.I) & 0.90 & & \\
\hline Los Olivos & $43(74.1)$ & $15(25.9)$ & I.3 (0.4-4.I) & 0.63 & & \\
\hline Independencia & $25(73.5)$ & $9(26.5)$ & I.3 (0.4-4.4) & 0.69 & & \\
\hline Comas & $13(68.4)$ & $6(3 I .6)$ & 1 & - & & \\
\hline Other & $54(69.2)$ & $24(30.8)$ & $\mathrm{n} / \mathrm{a}$ & & & \\
\hline \multicolumn{7}{|c|}{ Highest education attained } \\
\hline None/primary & $44(74.6)$ & $15(25.4)$ & $1.2(0.6-2.4)$ & 0.63 & & \\
\hline Secondary & $89(7 I .2)$ & $36(28.8)$ & I & - & & \\
\hline Technical & $28(70.0)$ & $12(30.0)$ & $0.9(0.4-2.1)$ & 0.88 & & \\
\hline University & $32(66.7)$ & $16(33.3)$ & $0.8(0.4-1.7)$ & 0.56 & & \\
\hline \multicolumn{7}{|l|}{ Occupation } \\
\hline None & $12(66.7)$ & $6(33.3)$ & $0.6(0.2-1.8)$ & 0.38 & & \\
\hline Homemaker & $72(76.6)$ & $22(23.4)$ & 1 & - & & \\
\hline Commerce & $16(66.7)$ & $8(33.3)$ & $0.6(0.2-1.6)$ & 0.32 & & \\
\hline Student & $22(78.6)$ & $6(21.4)$ & I.I (0.4-3.I) & 0.83 & & \\
\hline Driver/collector & $10(71.4)$ & $4(28.6)$ & $0.8(0.2-2.7)$ & 0.67 & & \\
\hline Construction & $6(54.6)$ & $5(45.5)$ & $0.4(0.1-1.3)^{\mathrm{a}}$ & $0.12^{\mathrm{a}}$ & - & \\
\hline Health care workers & $5(45.5)$ & $6(54.6)$ & $0.3(0.1-0.9)^{\mathrm{a}}$ & $0.04^{\mathrm{a}}$ & - & \\
\hline Other & $50(69.4)$ & $22(30.6)$ & $\mathrm{n} / \mathrm{a}$ & & & \\
\hline \multicolumn{7}{|l|}{ Socioeconomic status } \\
\hline No poverty & $181(74.2)$ & $63(25.8)$ & $3.8(1.7-8.5)^{\mathrm{a}}$ & $0.00 \mathrm{I}^{\mathrm{a}}$ & $3.8(1.6-8.9)$ & 0.002 \\
\hline Poverty & $12(42.9)$ & $16(57.1)$ & $\mathrm{I}$ & - & $\mathrm{I}$ & - \\
\hline \multicolumn{7}{|l|}{ Health insurance } \\
\hline None & $26(86.7)$ & $4(13.3)$ & $\mathrm{I}$ & - & $\mathrm{I}$ & - \\
\hline State health insurance & $123(65.8)$ & $64(34.2)$ & $0.3(0.1-0.9)^{\mathrm{a}}$ & $0.03^{\mathrm{a}}$ & $0.4(0.1-I . I)$ & 0.08 \\
\hline Social security (EsSalud) & $38(82.6)$ & $8(17.4)$ & $0.7(0.2-2.7)$ & 0.64 & $0.8(0.2-2.9)$ & 0.71 \\
\hline Other/Private & $6(66.7)$ & $3(33.3)$ & $0.3(0.1-1.8)^{\mathrm{a}}$ & $0.18^{a}$ & $0.2(0.04-1.4)$ & 0.12 \\
\hline \multicolumn{7}{|c|}{ Frequency of hospital visits } \\
\hline At least monthly & $43(59.7)$ & $29(40.3)$ & $0.5(0.3-1.0)^{\mathrm{a}}$ & $0.05^{\mathrm{a}}$ & $0.7(0.3-1.4)$ & 0.31 \\
\hline Every 3 months & $50(80.7)$ & $12(19.4)$ & $\mathrm{I} .4(0.6-3 . \mathrm{I})$ & 0.40 & $2.0(0.9-4.8)$ & 0.11 \\
\hline Twice a year & $38(69.1)$ & $17(30.9)$ & $0.8(0.4-1.6)$ & 0.47 & $0.7(0.3-1.6)$ & 0.45 \\
\hline Once a year or less & 62 (74.7) & $21(25.3)$ & I & - & $\mathrm{I}$ & - \\
\hline \multicolumn{7}{|c|}{ Self-reported perception of health status } \\
\hline Good/excellent & $129(72.9)$ & $48(27.1)$ & I & - & & \\
\hline Bad/really bad & $14(60.9)$ & $9(39.1)$ & $0.6(0.2-1.4)$ & 0.23 & & \\
\hline Regular & $50(69.4)$ & $22(30.6)$ & $0.8(0.5-1.5)$ & 0.59 & & \\
\hline
\end{tabular}

Notes: ${ }^{2}$ Variables with a $P<0.2$ in the bivariate analysis were included in the multivariate analysis. Adjusted $\mathrm{OR}$ and $95 \% \mathrm{Cl}$ are reported only for variables that remained significant or acted as confounders in the final multivariate model. Bold $p$-value $<0.05$ is statistically significant.

not significantly associated (Table 3 ). Socioeconomic status was the single determinant of health literacy (Table 4), and occupation and knowing someone with TB were the only determinants of TB knowledge (Table 3). Contrary to previous studies, level of education was not independently associated with TB knowledge or health literacy (Tables 3 and 4). 


\section{Health literacy}

The proportion of study participants scoring in the low health literacy range (29\%) was comparable to that reported in Buenos Aires, where $30 \%$ of hospital participants had low health literacy based on the results of the SAHLSA-50 assessment. ${ }^{12}$ In a similar study in Lima, $43 \%$ of patients receiving gynecological services had low health literacy. ${ }^{13}$ Another study, which utilized the Short version of Test of Functional Health Literacy in Adults, found that $32 \%$ of patients in Brazil had low health literacy. ${ }^{14}$ To our knowledge, no study in Latin America to date has utilized SAHL-S to evaluate health literacy. Outside of Latin America, a US study found that $36 \%$ of adults who performed an assessment involving 152 tasks had basic or below basic health literacy skills. ${ }^{11}$ This slightly higher proportion, when compared with the average of the four hospital studies in Latin America, may be partially explained by the fact that the US study utilized a nationally representative sample and those attending hospitals may have higher health literacy skills compared with the general population. Furthermore, health campaigns in Latin America are quite common and may increase exposure to and familiarity with medical terminology, which is an area tested in health literacy assessments like the SAHL-S and TOFHLA.

The strongest determinant of health literacy in our study was socioeconomic status: patients living in poverty were four times more likely to have low health literacy when compared with those who held higher economic status (Table 4). This is consistent with findings from the United States ${ }^{11}$ and United Kingdom $^{3}$ that demonstrate income constraints can limit access to health systems. We did not observe an association between the participant's level of education and health literacy (Table 4). We do not know why education and health literacy were not associated while socioeconomic status and health literacy were. We hypothesize that the knowledge and capacities related to health literacy may vary between people within the same level of education, and that this variation may be associated with socioeconomic status. For example, in Peru, people from impoverished households may have limited access to higher quality education compared with families with higher financial resources. Therefore, despite reporting the same level of education, there may be large variations in knowledge and skills among individuals, as a consequence of the quality of education they receive.

\section{TB knowledge}

TB knowledge was fair in our study population. Some important gaps in knowledge were observed as $29 \%$ of participants still reported very little or no TB in Peru. The most frequent spontaneous response to how participants thought one gets TB is "from being malnourished" (Table 2). And 20\% stated "better nutrition" is the cure for TB. This is an important risk factor in Peru, as there is evidence to suggest a dose-response relationship between degree of undernutrition and TB incidence. ${ }^{22}$ In Peru, milk and other nutrition are provided as adjuvants to TB treatment, though there is still insufficient data to prove whether this intervention actually improves treatment outcomes. ${ }^{23}$ More importantly, only $28 \%$ of participants could identify, without prompting, that being in contact with a TB patient is a risk factor for getting TB (Table 2). This is significant in that TB contacts may not even be aware that they need to seek medical attention, thus delaying diagnosis and increasing the risk of transmission and/or progression to active disease. In Lima, where there is a high incidence of TB and overcrowded transport systems, this is a public health crisis. ${ }^{24}$ Using public transportation is a known risk factor for TB in Lima and elsewhere, ${ }^{25}$ and it was reported as such by only $4.8 \%$ of study participants (Table 2 ). Although most participants indicated they would open the windows on public transportation, $17 \%$ still said closing windows "to avoid catching a cold" was better for their health as oppose to opening them for ventilation.

Public transport drivers and fare collectors are at high risk of TB from being exposed to overcrowded and less ventilated spaces. It was therefore concerning that this group also had the lowest TB knowledge scores of any occupation listed (Table 3). This is consistent with previous studies of transport workers in Peru, in which less than half of participants recognized any symptom of TB including cough (44\%), and only $41 \%$ perceived that being a driver or collector was a high-risk occupation for TB. ${ }^{26}$

When asked about TB symptoms, the most frequent spontaneous response was "cough" (70\%), but only $24 \%$ specified "cough for two weeks or more." Even though other symptoms were less frequently reported or recognized by our participants (Table 2), the constellation of several of these symptoms such as fever, weight loss, and fatigue is an important red flag that necessitates further workup and therefore is just as important and should be known by those at risk.

The factor most associated with high TB knowledge was direct experience with TB - defined as knowing someone who had TB (Table 3). This supports previous studies in Peru for both TB and other infectious diseases in which those most knowledgeable about a disease were those who knew someone who had been previously diagnosed, ${ }^{27,28}$ suggesting that person-person communication, advocacy, and community cohesion may play important roles in TB control. To increase case detection, health education needs to target risk groups and extend beyond the health care setting. More than half of participants in our study showed preference for television or 
social media as possible methods of receiving information related to TB. Novelas, popular TV shows in Latin America, could represent an avenue by which TB information is transmitted to the public in a cost-effective way, as most Peruvians in our study reported having access to a television in their household. Though, overall, less people reported having access to a computer or a smartphone, these could pave way for innovative technological interventions. While knowledge of TB is a crucial part of case detection, multiple other factors such as social stigma which may lead to lack of social support and access to health facilities also contribute to the under detection and diagnostic delay of TB in Lima. ${ }^{29}$

As opposed to studies showing associations between health literacy and knowledge of chronic diseases, ${ }^{8,9}$ we did not observe a significant association between health literacy and TB knowledge (Table 3). These results may reflect the impact of Peru's facility-based health talks, which are quite frequent, occur in patient waiting areas, and are led by nurse educators. The participants in our study may have been exposed to these educational activities which may have passively increased their TB knowledge. Furthermore, the fact that females made up $66 \%$ of our study population (Table 1) may have skewed the results; women in Peru tend to be the primary care givers and often visit health care facilities with their family members thus having a higher likelihood of engaging in or becoming exposed to these health talks.

\section{Limitations}

Our study had limited power to accurately determine associations between occupation and previous TB diagnosis. However, since we studied a representative sample of outpatients in a large referral hospital in Lima, our results may be generalizable to Lima and other urban areas in the region. Approximately $10.3 \%$ of our study participants lived in poverty (Table 1); this is consistent with official estimates for the districts where most of our participants reside: San Martin de Porres (10.5\%) and Los Olivos (9.8\%). ${ }^{30,31}$ By including hospital outpatients, we may have overestimated health literacy and TB knowledge. We enrolled participants who may be more "health aware" and have more access to formal health care. People without access to hospitals, either because they cannot afford it or prefer traditional medicines, were not represented in our study. Social desirability bias may have been present, and participants may have responded with answers thought to be more acceptable by health care workers. We minimized this potential bias by recording both spontaneous as well as elicited responses to items on TB risk and symptoms, and by training data collectors to maintain a neutral attitude when conducting the survey.

\section{Conclusion}

While health literacy was high in our study population, participants living in poverty had low health literacy scores, placing them at an increased risk of poor health outcomes, improper use of medications, and higher rates of emergency room visits and hospitalizations. ${ }^{32,33}$ Strategies to increase health literacy among impoverished communities in Lima could improve access and efficient use of health care services, and in the long run reduce health care costs. TB interventions should concentrate on high-risk areas (such as Lima) and among high-risk groups, including public transport drivers and users and contacts of TB patients. Increasing knowledge of TB symptoms and risk factors will reduce time to diagnosis, improve prognosis, and reduce risk of transmission which is critical in areas of high TB burden. ${ }^{34-37}$ Therefore, interventions such as novelas or smartphone technology and social media to promote TB education should be implemented and evaluated. TB health education programs for transport workers and users in Lima should also be pursued.

\section{Acknowledgments}

We acknowledge the support of Ms. Maribel Reyes and Ms. Maribel Riveros for their assistance with the study in Lima. This study was funded by the University of Alabama, Birmingham's Minority Health International Research Training grant no. T37 MD001448, from the National Institute on Minority Health and Health Disparities, National Institutes of Health, Bethesda, MD, USA. The abstract of this paper was presented at the 2018 National TB Conference as a poster presentation with interim findings.

\section{Disclosure}

The authors report no conflicts of interest in this work.

\section{References}

1. Nutbeam D. Health promotion glossary. Health Promot Int. 1998;13(4):349-364.

2. Institute of Medicine (US) Committee on Health Literacy, NielsenBohlman L, Panzer AM, Kindig DA, editors. Health Literacy: A Prescription to End Confusion. Washington, DC: National Academies Press; 2004.

3. Cunha GH, Galvão MT, Pinheiro PN, Vieira NF. Health literacy for people living with HIV/AIDS: an integrative review. Rev Bras Enferm. 2017;70(1):180-188.

4. Berkman ND, Dewalt DA, Pignone MP, et al. 87 Literacy and Health Outcomes: Summary. 2004 Jan. AHRQ Evidence Report Summaries. Rockville (MD): Agency for Healthcare Research and Quality (US); 1998-2005. Available from: https://www.ncbi.nlm.nih.gov/books/ NBK11942/. Accessed February 15, 2019.

5. Jones J, Rosaasen N, Taylor J, et al. Health literacy, knowledge, and patient satisfaction before kidney transplantation. Transplant Proc. 2016;48(8):2608-2614.

6. Son YJ, Yu HY. Influence of health literacy on HRQoL in patients after PCI. West J Nurs Res. 2016;38(12):1611-1626. 
7. Juzych MS, Randhawa S, Shukairy A, Kaushal P, Gupta A, Shalauta N. Functional health literacy in patients with glaucoma in urban settings. Arch Ophthalmol. 2008;126(5):718-724.

8. Williams MV, Baker DW, Parker RM, Nurss JR. Relationship of functional health literacy to patients' knowledge of their chronic disease. A study of patients with hypertension and diabetes. Arch Intern Med. 1998;158(2):166-172.

9. Gazmararian JA, Williams MV, Peel J, Baker DW. Health literacy and knowledge of chronic disease. Patient Educ Couns. 2003;51(3):267-275.

10. Parker RM, Kindig DA. Beyond the Institute of medicine health literacy report: are the recommendations being taken seriously? J Gen Intern Med. 2006;21(8):891-892.

11. Greenberg E, Jin Y. 2003 National Assessment of Adult Literacy: Public-Use Data File User's Guide. Washington, DC: National Center for Education Statistics; 2007.

12. Konfino J, Mejia R, Majdalani MP, Perez-Stable EJ. Alfabetizacion en Salud en Pacientes Que Asisten a un Hospital Universitario. Medicina. 2009;69(6):631-634.

13. Vílchez-Román C, Quiliano-Terreros R, Canales-Negrón R, ChiroqueSolano R. Factores demográficos asociados Con El conocimientosobresalud en Unamuestra de mujeres de la ciudad de lima (Perú) en 2008: resultados del cuestionario SAHLSA-50. [Demographic factors associated with health literacy in a sample of females residing in Lima, Peru in 2008: results from the SAHLSA-50 test] Revista Colombiana de Obstetricia y Ginecología. 2009;60(3):223-229.

14. Carthery-Goulart MT, Anghinah R, Areza-Fegyveres R, et al. Performance of a Brazilian population on the test of functional health literacy in adults. Rev Saúde Pública. 2009;43(4):631-638.

15. WHO. Global Tuberculosis Report 2017. Geneva: World Health Organization; 2017.

16. Organización Panamericana de la Salud/Organización Mundial de la Salud. Situacion de la tuberculosis en LAS Americas 2016. 2017. Available from: https://www.paho.org/hq/dmdocuments/2017/2017cha-hoja-info-situacion-tb-americas.pdf. Accessed February 15, 2019.

17. Castro-Sánchez E, Chang PWS, Vila-Candel R, Escobedo AA, Holmes $\mathrm{AH}$. Health literacy and infectious diseases: why does it matter? Int $J$ Infect Dis. 2016;43:103-110.

18. Farmer P, Bayona J, Becerra M. The dilemma of MDR-TB in the global era. Int J Tuberc Lung Dis. 1998;2(11):869-876.

19. Lima: ESNPCT-MINSA. Estrategia Sanitaria Nacional de Prevención y Control de la Tuberculosis. Situación epidemiológica de la tuberculosis - Perú. Al primer semestredel2012. 2012. Available from: http://www. tuberculosis.minsa.gob.pe/portaldpctb/recursos/20180605122521.pdf. Accessed February 15, 2019.

20. Ministerior de Economia y Finanzas. Propuesta Metodologica Para Identificar a Los Potenciales Beneficiarios De los Programas Sociales en el Peru. Direccion General de Asuntos Economicos y Sociales Sistema de Focalizacion de Hogares- SISFOH. 2009. Available from: http:// www.bcrp.gob.pe/docs/Publicaciones/Documentos-de-Trabajo/2010/ Documento-de-Trabajo-06-2010.pdf. Accessed February 15, 2019.

21. Lee SY, Stucky BD, Lee JY, Rozier RG, Bender DE. Short assessment of health Literacy-Spanish and English: a comparable test of health literacy for Spanish and English speakers. Health Serv Res. 2010;45(4):1105-1120.
22. Lönnroth K, Williams BG, Cegielski P, Dye C. A consistent log-linear relationship between tuberculosis incidence and body mass index. Int J Epidemiol. 2010;39(1):149-155.

23. Grobler L, Nagpal S, Sudarsanam TD, Sinclair D. Nutritional supplements for people being treated for active tuberculosis. Cochrane Database Syst Rev. 2016;52(3):CD006086.

24. Zamudio C, Krapp F, Choi HW, et al. Public transportation and tuberculosis transmission in a high incidence setting. PLoS One. 2015;10(2): $\mathrm{e} 0115230$

25. Horna-Campos OJ, Bedoya-Lama A, Romero-Sandoval NC, MartínMateo M. Risk of tuberculosis in public transport sector workers, Lima, Peru. Int J Tuberc Lung Dis. 2010;14(6):714-719.

26. Lukac D, Garaycochea O, Taype-Rondan A, Luque Bustamante L, Mujica-Vasquez A, Zamora D. Conocimientos y percepciones sobre tuberculosis pulmonar en trabajadores del transporte público en Lima, Perú [Knowledge and perception about tuberculosis among public transport workers in Lima, Peru]. Medwave. 2016;16(10):e6629.Spanish.

27. Westerlund EE, Tovar MA, Lönnermark E, Montoya R, Evans CA. Tuberculosis-related Knowledge is associated with patient outcomes in shantytown residents; results from a cohort study, Peru. $J$ Infect. 2015;71(3):347-357.

28. Paz-Soldán VA, Morrison AC, Cordova Lopez JJ, et al. Dengue knowledge and preventive practices in Iquitos, Peru. Am J Trop Med Hyg. 2015;93(6):1330-1337.

29. Duarte R, Lönnroth K, Carvalho C, Lima F, Carvalho ACC, Muñoz-Torrico M, Centis R. Tuberculosis, social determinants and co-morbidities (including HIV). Pulmonology. 2018;24(2):115-119.

30. Instituto Nacional de Estadística E Informática (INEI). Mapa de la Pobreza Distrital 2013.2013. available from: https://www.inei.gob.pe/ media/MenuRecursivo/publicaciones_digitales/Est/Lib1261/Libro.pdf. Accessed February 15, 2019

31. Rivas-Legua P. Pobreza en Lima: los distritos con máscarencias [MAPA]. Diario El Comercio. 2015. Available from: https://enriqueremy.lamula.pe/2016/09/08/la-pobreza-urbana-en-el-peru/eremy/. Accessed February 15, 2019.

32. Marais BJ, Hesseling AC, Cotton MF. Poverty and tuberculosis: is it truly a simple inverse linear correlation? Eur Respir J. 2009;33(4):943-944.

33. Office of Disease Prevention and Health Promotion. National Action Plan to Improve Health Literacy. Washington, DC: U.S. Department of Health and Human Services. 2010.

34. Paz-Soldan VA, Alban RE, Dimos Jones C, Powell AR, Oberhelman RA. Patient reported delays in seeking treatment for tuberculosis among adult and pediatric TB patients and TB patients co-infected with HIV in Lima, Peru: a qualitative study. Front Public Health. 2014;2(1):281.

35. Saifodine A, Gudo PS, Sidat M, Black J. Patient and health system delay among patients with pulmonary tuberculosis in Beira City, Mozambique. BMC Public Health. 2013;13(1):559.

36. Makwakwa L, Sheu ML, Chiang CY, Lin SL, Chang PW. Patient and Heath system delays in the diagnosis and treatment of new and retreatment pulmonary tuberculosis cases in Malawi. BMC Infect Dis. 2014;14(1):132.

37. Biya O, Gidado S, Abraham A. Knowledge, care-seeking behavior, and factors associated with patient delay among newly-diagnosed pulmonary tuberculosis patients, Federal Capital Territory, Nigeria, 2010. Pan Afr Med J. 2014;18(Suppl 1):6.
Research and Reports in Tropical Medicine

\section{Publish your work in this journal}

Research and Reports in Tropical Medicine is an international, peerreviewed, open access journal publishing original research, case reports, editorials, reviews and commentaries on all areas of tropical medicine, including: Diseases and medicine in tropical regions; Entomology; Epidemiology; Health economics issues; Infectious disease; Laboratory
Dovepress

science and new technology in tropical medicine; Parasitology; Public health medicine/health care policy in tropical regions; and Microbiology. The manuscript management system is completely online and includes a very quick and fair peer-review system. Visit http://www.dovepress. com/testimonials.php to read real quotes from published authors. 